\title{
Ezetimibe Enhances Macrophage-to-Feces Reverse Cholesterol Transport in Golden Syrian Hamsters Fed a High-Cholesterol Diet
}

\author{
Fatima Kasbi-Chadli, ${ }^{1}$ Morgan Treguier, ${ }^{1}$ François Briand, Thierry Sulpice, \\ and Khadija Ouguerram
}

INRAe, UMR 1280, Physiopathologie des Adaptations Nutritionnelles, CHU Hôtel-Dieu, Nantes, France (F.K.-C., M.T., K.O.) and Physiogenex SAS, Prologue Biotech, Rue Pierre et Marie Curie, Laboratoryège-Innopole, France (F.B., T.S.)

Received April 23, 2020; accepted August 4, 2020

\begin{abstract}
The aim of this work was to evaluate reverse cholesterol transport (RCT) in hamster animal model expressing cholesterol ester transfer protein under a high-cholesterol diet (HF) supplemented with ezetimibe using primary labeled macrophages. We studied three groups of hamsters ( $n=8 /$ group) for 4 weeks: 1 ) chow diet group: Chow, 2) high-cholesterol diet group: HF, and 3) HF group supplemented with $0.01 \%$ of ezetimibe: $\mathrm{HF}+0.01 \%$ Ezet. After intraperitoneal injection of ${ }^{3} \mathrm{H}$-cholesterol-labeled hamster primary macrophages, we measured the in vivo macrophage-tofeces RCT. HF group exhibited an increase of triglycerides (TGs), cholesterol, and glucose in plasma and higher TG and cholesterol content in liver $(P<0.01)$ compared with Chow group. Ezetimibe induced a significant decrease in plasma cholesterol with a lower low-density lipoprotein (LDL) and very LDL cholesterol $(P<0.001)$ and in liver cholesterol $(P<0.001)$ and TG $(P<0.01)$ content compared with HF. In vivo RCT essay showed an increase of tracer level in plasma and liver $(P<0.05)$ but not in feces in HF compared with Chow group. The amount of labeled total sterol and cholesterol in liver and feces was significantly reduced $(P<$ $0.05)$ and increased $(P=0.05)$, respectively, with ezetimibe treatment. No significant increase was obtained for labeled feces
\end{abstract}

bile acids in $\mathrm{HF}+0.01 \%$ Ezet compared with HF. Ezetimibe decreased stearoyl-CoA desaturase-1 gene expression and increased scavenger receptor type B class $1(P<0.05)$ in liver but did not affect Niemann-Pick C1-like 1 nor ATP-binding cassette (ABC) G5 and ABCG8 expression in jejunum. In conclusion, ezetimibe exhibited an atheroprotective effect by enhancing $\mathrm{RCT}$ in hamster and decreasing LDL cholesterol. Our findings also showed a hepatoprotective effect of ezetimibe by decreasing hepatic fat content.

\section{SIGNIFICANT STATEMENT}

This work was assessed to determine the effect of ezetimibe treatment on high-cholesterol diet-induced disturbances and especially the effect on reverse cholesterol transport in animal model with cholesterol ester transfer protein activity and using labeled primary hamster macrophages. We were able to demonstrate that ezetimibe exhibited an atheroprotective effect by enhancing reverse cholesterol transport and by decreasing lowdensity lipoprotein cholesterol in hamster. We also showed a hepatoprotective effect of ezetimibe by decreasing hepatic fat content.

\section{Introduction}

Metabolic syndrome is a cluster of metabolic disturbances that increase cardiovascular disease. Dyslipidemia is a common disturbance in metabolic syndrome (Ginsberg et al., 2006). Cholesterol homeostasis is a balance of cholesterol synthesis, intestinal absorption, and bile acids elimination. The current treatment of hypercholesterolemia is statins, which lower the hepatic cholesterol synthesis by inhibiting 3-hydroxy-3-methylglutaryl-CoA enzyme activity and then accelerate hepatic low-density lipoprotein (LDL) uptake. Active hepatic synthesis of cholesterol and LDL uptake are also modulated by the amount of absorbed cholesterol in the

This research received external funding from French National Research Agency (ANR).

${ }^{1}$ F.K.-C. and M.T. contributed equally as co-first authors.

https://doi.org/10.1124/jpet.120.000062. small intestine. Ezetimibe is known to inhibit cholesterol absorption via Niemann-Pick C1-like 1 (NPC1L1)-mediated cholesterol absorption at the brush border of the intestine (Altmann et al., 2004). NPC1L1 has been identified as the main transporter of intestinal cholesterol and has been shown to be a target of ezetimibe (Garcia-Calvo et al., 2005). Ezetimibe is an effective lowering lipid drug (Jacobson et al., 2015). A large clinical study in individuals aged $\geq 75$ years with elevated LDL cholesterol showed that ezetimibe prevented cardiovascular events by decreasing LDL cholesterol (Ouchi et al., 2019).

Ezetimibe enhances macrophage-to-feces reverse cholesterol transport (RCT) in mice as shown by in vivo RCT experiments (Briand, 2010; Wang et al., 2014). In the present study, hamsters have been used as an alternate model with lipoprotein metabolism close to humans to determine the impact of ezetimibe treatment on the efficacy of RCT. Contrary to more

ABBREVIATIONS: ABC, ATP-binding cassette; CETP, cholesterol ester transfer protein; Ezet, ezetimibe; HDL, high-density lipoprotein; HF, highcholesterol diet; LCAT, lecithin cholesterol acyl transferase; LDL, low-density lipoprotein; NPC1L1, Niemann-Pick C1-like 1; RCT, reverse cholesterol transport; SCD1, stearoyl-CoA desaturase-1; SR-B1, scavenger receptor type B class 1; TG, triglyceride; VLDL, very-low-density lipoprotein. 
classic protocols using murine cells (Tchoua et al., 2008; Castro-Perez et al., 2011), we have studied (Kasbi-Chadli et al., 2013) the RCT in hamster using labeled primary hamster macrophages. The use of primary hamster macrophages avoids the cross-species inflammatory reaction that could potentially lead to an immunologic targeted destruction of macrophages by using J774 murine cells. Moreover, these cells do not express apolipoprotein E gene (Bernard et al., 1991) involved in the removal of excess cholesterol from macrophage foam cells (Mazzone and Reardon, 1994). Few studies with regards to ezetimibe have been conducted using hamster, and only one of them focused on the cholesterol flux from peripheral cells to the liver to further excretion into bile and feces (Uto-Kondo et al., 2014) using labeled primary hamster macrophages. The hamster model, unlike the mice model, is a model that expressed cholesterol ester transfer protein (CETP), which plays an important role in RCT. It is established that plasma cholesterol concentrations and cholesterol distribution among lipoproteins are affected by ezetimibe in hamster (van Heek et al., 2001). It has never been studied whether these changes in the metabolism lipoproteins induced by ezetimibe observed in hamster affect lipid and glucose metabolic disturbances.

To address this question, we characterized the effects of a high-cholesterol diet supplemented with $0.01 \%$ ezetimibe in Golden Syrian hamsters and assessed in vivo macrophage-tofeces RCT using labeled primary macrophages as well as the effect of ezetimibe on lipid and glucose metabolic disturbances.

\section{Material and Methods}

\section{Ethics Statement}

The experimental protocol was approved by the local Animal Use and Care Advisory Committee (Bretagne-Pays de la Loire committee) and adhered to European Union guidelines. Animals were sacrificed by intracardiac injection of lethal dose of pentobarbital. All animal trials were carried out under isoflurane anesthesia.

\section{Animals and Diets}

Male hamsters (R. Janvier, France) were housed in plastic cages (two hamsters/cage) containing wood shavings and maintained in a room with a 12-hour light cycle with free access to food and water. Animals (82-99 g) were adapted to these conditions and fed a rodent chow diet (A04 rodent diet; Safe Diets, France) for 1 week. This rodent chow diet was defined as the control diet.

Hamsters were then kept on the control diet $(n=8)$, diet rich in cholesterol ( $0.3 \%$ of cholesterol) $(n=8)$, or high-cholesterol diet supplemented with $0.01 \%$ ezetimibe $(n=8)$ during 4 weeks. The groups with high-cholesterol diet received 10\% fructose in drinking water. Hamsters were fasted overnight, and slight isoflurane anesthesia was used to collect blood by retro-orbital bleeding to measure plasma lipids. After sacrifice, hamsters were exsanguinated, and liver was harvested, flash-frozen in liquid nitrogen, and stored at $-80^{\circ} \mathrm{C}$ until analysis. Blood glucose was monitored using a glucometer (Roche Diagnostics, France). Three other separate sets of hamsters were fed the chow, high cholesterol or high cholesterol + ezetimibe diet over 4 weeks, and were used for determination of in vivo triglyceride production ( $n=8$ per group) and in vivo macrophage-to-feces RCT ( $n=8$ per group), as described below.

\section{Biochemical Analysis}

To determine plasma lipids, hamsters were fasted overnight (18 hours), and blood was collected by retro-orbital under isoflurane anesthesia. Blood was then centrifugated $\left(4^{\circ} \mathrm{C}, 10\right.$ minutes, $\left.3000 \mathrm{~g}\right)$ to separate plasma. Triglyceride and total cholesterol were measured using commercial kits (Biomérieux, France). Lipoproteins were separated using fast protein liquid chromatography (AKTA FPLC SYSTEM; GE Healthcare). CETP activity was assayed by a commercial kit (RoarBiomedical, NY). Evaluation of Lecithin cholesterol acyl transferase (LCAT) activity was performed by the measurement of the decrease of free cholesterol after 1 hour of plasma incubation at $37^{\circ} \mathrm{C}$. This decrease is expressed as the percentage of free cholesterol transformed into cholesteryl ester by hour (Nagasaki and Akanuma, 1977).

Intraperitoneal Glucose Tolerance Test. An intraperitoneal glucose tolerance test was performed after hamsters were unfed for 18 hours. Eight hamsters from each group received $2 \mathrm{~g}$ of glucose per kilogram body weight intraperitoneally. Blood samples were obtained from tail vein at 0 (fasting), 15, 20,30,60, and 120 minutes, and glycemia was measured immediately using fresh blood (glucometer: Accu-Chek active; Roche Diagnostics, Mannhein, Germany). Areas under the curve were calculated by the trapezoid rule from the glucose measurements at 0 (fasting), 15, 30, 60, 120, and 180 minutes (milligram $\times$ minute per deciliter).

Measurement of In Vivo Hepatic Secretion of Triglycerides. The effect of ezetimibe on the hepatic triglyceride (TG) secretion was determined using Tyloxapol, a nonionic surfactant (Triton WR1339) that inhibits lipoprotein lipase (Schotz et al., 1957). Hamsters were anesthetized with isoflurane, and blood samples were collected from the eye orbital venous plexus. Blood samples were taken at baseline just before peritoneal injection of Tyloxapol and then at 30 minutes (t30), 2 hours (t120), and 3 hours (t180). The first 30 minutes after Tyloxapol injection are required to detergent equilibration and initiation of lipoprotein accumulation (Millar et al., 2002). Plasma TG accumulation from t30 to t180 after Tyloxapol injection is proportional to very-low-density lipoprotein (VLDL) TG secretion (Siri et al., 2001). Hepatic TG production rate for individual hamsters was therefore calculated using linear increment between 30 minutes and 3 hours.

\section{Isolation of Peritoneal-Elicited Macrophages}

Isolation of peritoneal macrophages was described in our previous study (Treguier et al., 2011). Briefly, hamsters were injected intraperitoneally with $3 \%$ Brewer thioglycollate medium (Sigma). Three days after injection, hamsters were sacrificed, and macrophages were collected by peritoneal washing with ice-cold PBS and then centrifuged. The macrophages were suspended in $5 \mathrm{ml}$ of red blood cell lysis buffer and incubated in ice for 15 minutes. After centrifugation, the macrophages were collected and suspended in RPMI culture medium supplemented with $10 \%$ FBS (Gibco). The cells were then plated in flasks in RPMI complete medium and allowed to adhere; nonadherent cells were removed.

\section{In Vivo Macrophage-to-Feces Reverse Cholesterol Transport}

Hamster macrophages were radiolabeled as described previously (Treguier et al., 2011). Briefly, macrophages were loaded with ${ }^{3} \mathrm{H}$-cholesterol-labeled acetylated LDL. Cells were pelleted by centrifugation and suspended in PBS before hamster injection ( $n=6$ /group). Hamsters were then housed in individual cage. Blood was drawn at 48 and 72 hours, and radioactivity was counted in a liquid scintillation counter. After hamster sacrifice, liver was harvested to assess the radioactivity uptake.

The total feces collected over 72 hours was homogenized to extract the ${ }^{3} \mathrm{H}$-cholesterol and ${ }^{3} \mathrm{H}$-bile acid fractions as we have previously described (Treguier et al., 2011). The extracts were treated to determine radioactivity. Results were expressed as a percent of the radioactivity injected recovered in plasma, liver, and feces. The volume of plasma was estimated as $3.5 \%$ of the body weight. 
TABLE 1

Body weight and plasma biochemical parameters after overnight fast in hamsters fed a control chow diet, a high-cholesterol diet, or a high-cholesterol diet supplemented with $0.01 \%$ ezetimibe over $4 \mathrm{wk}$

Mean \pm S.E.M., $n=8$ per group.

\begin{tabular}{|c|c|c|c|}
\hline & Chow & High-Fat Diet (HF) & High-Fat Diet $+0.01 \%$ Ezetimibe $(\mathrm{HFe})$ \\
\hline Body weight (g) & $105.9 \pm 2.48$ & $104.7 \pm 3.21$ & $103.9 \pm 1.81$ \\
\hline Fasted blood glucose $(\mathrm{mg} / \mathrm{dl})$ & $106.5 \pm 4.64$ & $137.88 \pm 8.84^{* *}$ & $139.75 \pm 5.98^{* * *}$ \\
\hline Total cholesterol $(\mathrm{g} / \mathrm{l})$ & $1.35 \pm 0.05$ & $2.65 \pm 0.19 * * *$ & $2.04 \pm 0.11^{* * *} \dagger$ \\
\hline HDL cholesterol $(\mathrm{g} / \mathrm{l})$ & $1.22 \pm 0.1$ & $1.57 \pm 0.1^{*}$ & $1.48 \pm 0.03 * *$ \\
\hline HDL cholesterol/total cholesterol & $0.96 \pm 0.05$ & $0.61 \pm 0.07 * * *$ & $0.75 \pm 0.05^{* *}$ \\
\hline Free cholesterol $(\mathrm{mg} / \mathrm{dl})$ & $25.3 \pm 0.94$ & $48.17 \pm 3.13^{* *}$ & $41.87 \pm 1.79 * * *$ \\
\hline Triglycerides $(g / l)$ & $1.22 \pm 0.12$ & $2.7 \pm 0.40 * *$ & $3.15 \pm 0.29$ \\
\hline Free fatty acids $(\mathrm{mmol} / \mathrm{l})$ & $0.51 \pm 0.06$ & $0.47 \pm 0.05$ & $0.58 \pm 0.03$ \\
\hline CETP activity $(\mathrm{pmol} / \mathrm{h}$ per $\mu \mathrm{l})$ & $26.08 \pm 1.16$ & $29.19 \pm 2.14$ & $26.78 \pm 1.29$ \\
\hline LCAT activity (nmol/h per ml) & $350.65 \pm 28.102$ & $617.29 \pm 47.54^{* * *}$ & $674.39 \pm 51.57^{* * *}$ \\
\hline
\end{tabular}

${ }^{*} P<0.05 ; * * P<0.01 ; * * * P<0.001$ vs. Chow, $+P<0.05$ vs. HF.

\section{RNA Extraction and Gene Expression Analysis}

After homogenization of liver samples, mRNA was extracted using Trizol reagent (Invitrogen, France). Reverse-transcription polymerase chain reaction analysis was assessed as follows: $1 \mu \mathrm{g}$ of total RNA was reverse-transcribed using MML-V reverse transcriptase (Promega, France). Real-time quantitative polymerase chain reaction was performed on the 7000 Sequence Detection System with SYBR green MESAGREEN Master Mix Plus (Eurogentec, Angers, France). All reactions were duplicates. Cyclophilin was used as a reference. The expression of CYP7A1, SR-B1, LDLr (LDL receptor), and ATP-binding cassette (ABC) A1 was measured in liver (eight hamsters/group). The expression of NPC1L1, ABCG5, and ABCG8 was measured in intestine. Primers sequences are available on request.

\section{Determination of Hepatic Lipid Content}

Lipids were extracted from $30 \mathrm{mg}$ of hepatic tissue using Folch method (Folch et al., 1957). The tissue was homogenized with chloroform/methanol (2/1) and then centrifuged to recover the liquid phase. The solvent was washed with saline solution and centrifuged. The chloroform phase was evaporated, and lipids were suspended in ethanol. Triglyceride and cholesterol concentration was assessed using commercial kits (TG: PAP 150, cholesterol RTU; Biomérieux, Marcy-l'Etoile, France) ( $n=8$ /group).

\section{Statistical Analysis}

The statistical significance of differences between groups was evaluated using ANOVA test followed by two-tailed student's $t$ test for unpaired comparisons. $P<0.05$ was considered significant. Values are presented as mean \pm S.E.M.

\section{Results}

Ezetimibe Did Not Affect Weight and Fasted Blood Glucose and Glucose Tolerance, but It Decreased Plasma Cholesterol by Decreasing VLDL and LDL Cholesterol. No change was observed for body weight among groups. Blood fasted glucose was higher in groups under highcholesterol diet compared with chow control group with no difference between $\mathrm{HF}$ and $\mathrm{HF}+0.01 \%$ Ezet (Table 1 ). The measurement of areas under the curve after intraperitoneal glucose tolerance test did not show any difference in glucose tolerance among the three groups (unpublished data).

High-cholesterol diet significantly increased plasma cholesterol $(P<0.001)$, TGs $(P<0.001)$, and LCAT activity
$(P<0.05)$. Ezetimibe supplementation did not affect TG concentration and LCAT activity but significantly lowered plasma cholesterol concentration in $\mathrm{HF}+0.01 \%$ Ezet compared with HF $(P<0.005)$ (Table 1$)$. The cholesterol lipoprotein profile showed a significant decrease in VLDL $(P<0.05)$ and LDL $(P<0.001)$ cholesterol in HF $+0.01 \%$ Ezet compared with HF $(P<0.005)$ (Fig. 1). No effect was observed on CETP activity (Table 1).

Ezetimibe Lowered Hepatic Lipid Accumulation but Did Not Affect Hepatic VLDL TG Secretion. Although the total liver weight was significantly higher in HF compared with Chow group $(P<0.01)$ this parameter is normalized in $\mathrm{HF}+0.01 \%$ Ezet group compared with $\mathrm{HF}$ group $(P<0.005)$. The measurement of lipid content in liver showed a significant increase in TG and cholesterol in HF group compared with Chow group $(P<0.005)$. Ezetimibe treatment canceled the cholesterol accumulation in liver $(P<$ $0.001)$ and decreased TG content in liver in $\mathrm{HF}+0.01 \%$ Ezet group compared with HF group $(P<0.01$, Fig. 2$)$. The measure of hepatic TG secretion did not show a significant difference among groups even between $\mathrm{HF}$ and Chow group (Fig. 3).

Ezetimibe Promoted Fecal Cholesterol Excretion without Effect on Bile Acids Excretion. To assess whether RCT is affected in vivo, control Chow, HF, and HF + $0.01 \%$ Ezet hamsters were injected with ${ }^{3} \mathrm{H}$-cholesterol-labeled hamster primary macrophages. Fig. 4A showed higher plasma ${ }^{3} \mathrm{H}$-tracer appearance at 24 hours in HF compared with control Chow group. At 72 hours, plasma ${ }^{3} \mathrm{H}$-tracer appearance was lower in $\mathrm{HF}+0.01 \%$ Ezet group compared with Chow group $(P=0.02)$. No change was observed at 48 hours after macrophage injection among the three groups (Fig. 4A).

Tracer recovery in liver was higher in HF group compared with control chow diet $(P<0.05)$, whereas HF $+0.01 \%$ Ezet group exhibited less ${ }^{3} \mathrm{H}$-cholesterol accumulation compared with $\mathrm{HF}(P=0.05)$ (Fig. $4 \mathrm{~B})$.

No difference was measured between $\mathrm{HF}$ and control for fecal cholesterol and bile acids excretion (Fig. 4C). A higher ${ }^{3} \mathrm{H}$-tracer was measured in fecal total sterols in $\mathrm{HF}+0.01 \%$ Ezet compared with both $\mathrm{HF}$ and control chow $\operatorname{diet}(P<0.05)$. This increase in total sterol excretion was mainly related to an increase in fecal cholesterol since no significant effect of ezetimibe on tracer recovery in fecal bile acids was observed among groups (Fig. 4C). 
A

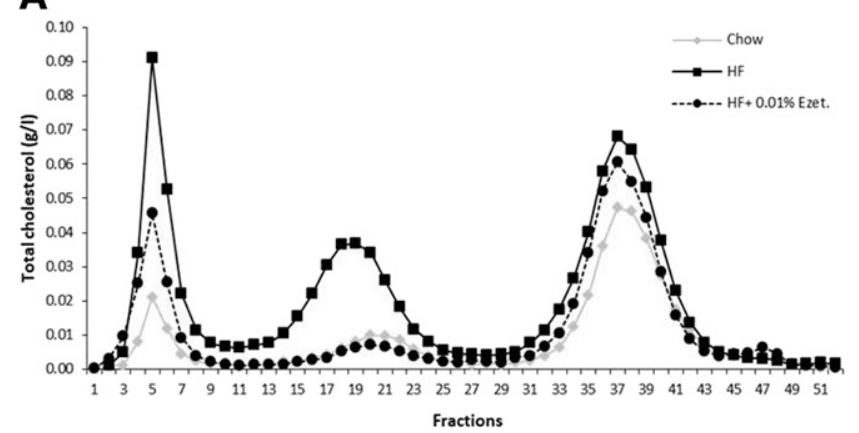

Fractions
B

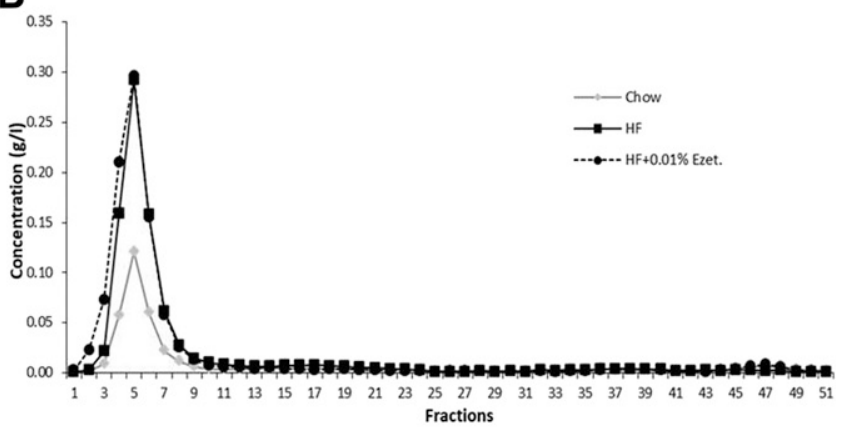

C

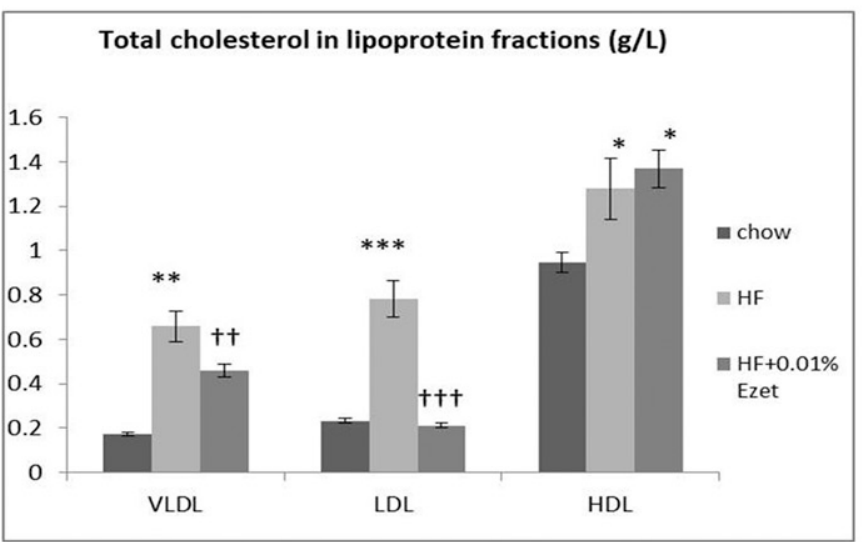

Fig. 1. Distribution of cholesterol (A) and triglycerides (B) in the plasma of overnight-fasted hamsters after a 4-week chow diet, high-cholesterol diet, or high-cholesterol diet $+0.01 \%$ ezetimibe analyzed by gel filtration chromatography (pooled plasma). Values are mean \pm S.E.M. $(n=8$ per group, *** $P<0.001$ vs. chow-fed hamsters).

Cholesterol Metabolism Gene Expression Is Affected by Ezetimibe Treatment. In liver, the measurement of gene expression showed a decrease in gene expression of LDLr and ABCA1 in HF and HF $+0.01 \%$ Ezet groups compared with chow control $(P<0.05)$. The gene expression of SR-B1 was lower in HF compared with control $(P<$ $0.05)$. Treatment with ezetimibe normalized the expression of SR-B1 $(P<0.01)$ without affecting LDLr and ABCA1 (Fig. 5A).

Stearoyl-CoA desaturase-1 (SCD1) and CYP7A1 gene expression was higher in HF group compared with Chow group $(P<0.001)$. Treatment with ezetimibe significantly decreased SCD1 $(P<0.005)$ expression and had no effect on CYP7A1
(Fig. 5A). In intestine, NPC1L1, ABCG5, and ABCG8 expression did not differ among the three groups (Fig. 5B).

\section{Discussion}

The process of RCT consists of the delivery of cholesterol from peripheral tissues to the liver followed by its secretion into the bile and, subsequently, its final elimination in the feces. RCT in cholesterol-loaded macrophages from atherosclerotic plaques plays a critical role in disease prevention by mediating the transfer of this cholesterol to the liver (or intestine) for disposal (Cuchel and Rader, 2006). It was reported that the absorption of cholesterol from the intestine
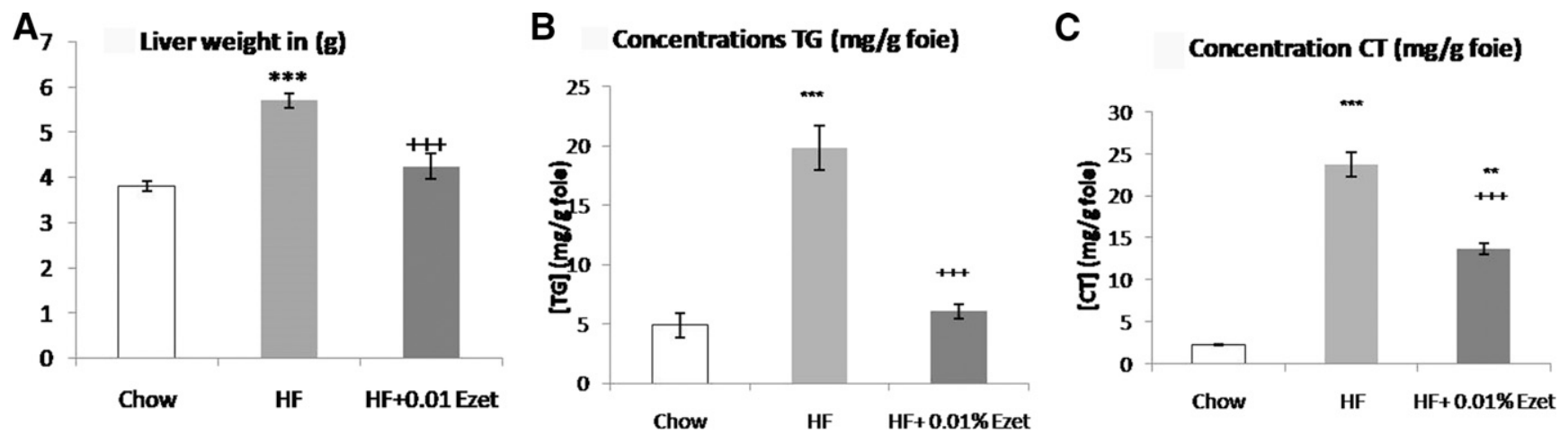

Fig. 2. Liver weight (in gram) and hepatic total cholesterol and triglycerides levels (in milligrams per gram of liver) in hamsters fed a 4 -week chow diet, high-cholesterol diet, or high-cholesterol $+0.01 \%$ Ezet diet. Values are mean \pm S.E.M. $(n=8$ per group, $* * * P<0.001$ vs. chow-fed hamsters). 

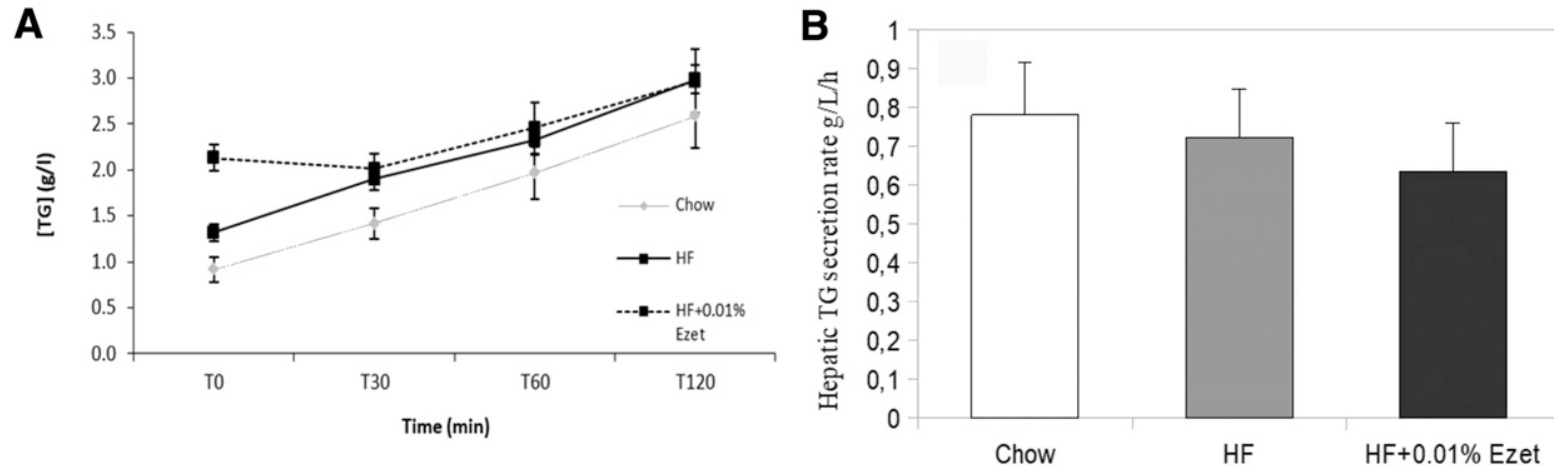

Fig. 3. After a 4-week chow diet, high-fat diet, or high-cholesterol $+0.01 \%$ Ezet diet, Tyloxapol ( $500 \mathrm{mg} / \mathrm{kg}$ body weight) was administered intravenously to overnight-fasted hamsters. Hamsters were bled at times $0,30,60,90$, and 120 minutes after injection. Plasma samples from each time point were used to determine plasma triglycerides levels over time (A). Triglyceride production (B) was calculated by subtracting the values at time 30 minutes from those at time 120 minutes in chow-, HF-, or HF + 0.01\%Ezet-fed hamsters.

is an important determinant of macrophage-to-feces RCT (Sehayek and Hazen, 2008; Briand et al., 2009).

The present study investigated the effect of ezetimibe on reverse cholesterol transport in hamster, an animal model with cholesterol metabolism close to that of human, by using primary labeled macrophages. The results demonstrated that ezetimibe treatment significantly reduced the plasma cholesterol by decreasing VLDL and LDL cholesterol and promoted fecal cholesterol excretion in hamsters fed with high-cholesterol diet compared with control hamsters. Moreover, ezetimibe decreased triglycerides and cholesterol content in liver, indicating favorable effects on liver steatosis. This result was associated with a significant reduction in mRNA levels of SCD1 in the liver. Ezetimibe treatment did not improve higher glycemia induced by high-cholesterol diet.

In our study, high-cholesterol diet associated with fructose in drinking water increased fasting blood glucose in $\mathrm{HF}$ and $\mathrm{HF}+0.01 \%$ Ezet groups compared with control, and no difference was observed in presence of ezetimibe treatment. This result was also obtained in other study in hamsters fed high-fat diet and supplemented with ezetimibe, in which ezetimibe improved lipid profile without affecting plasma
A

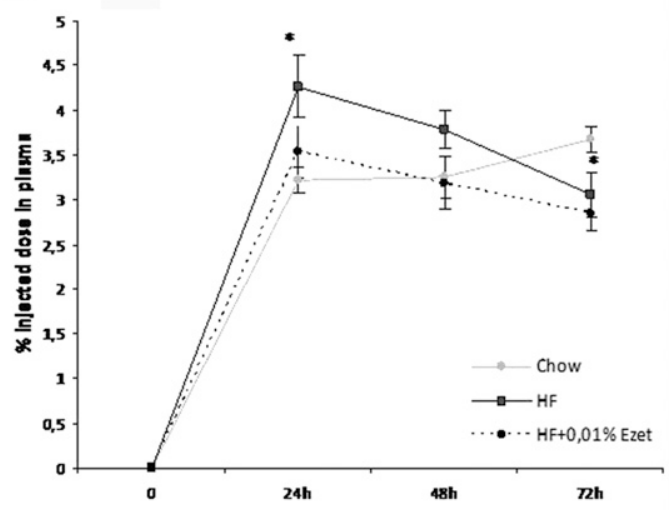

B
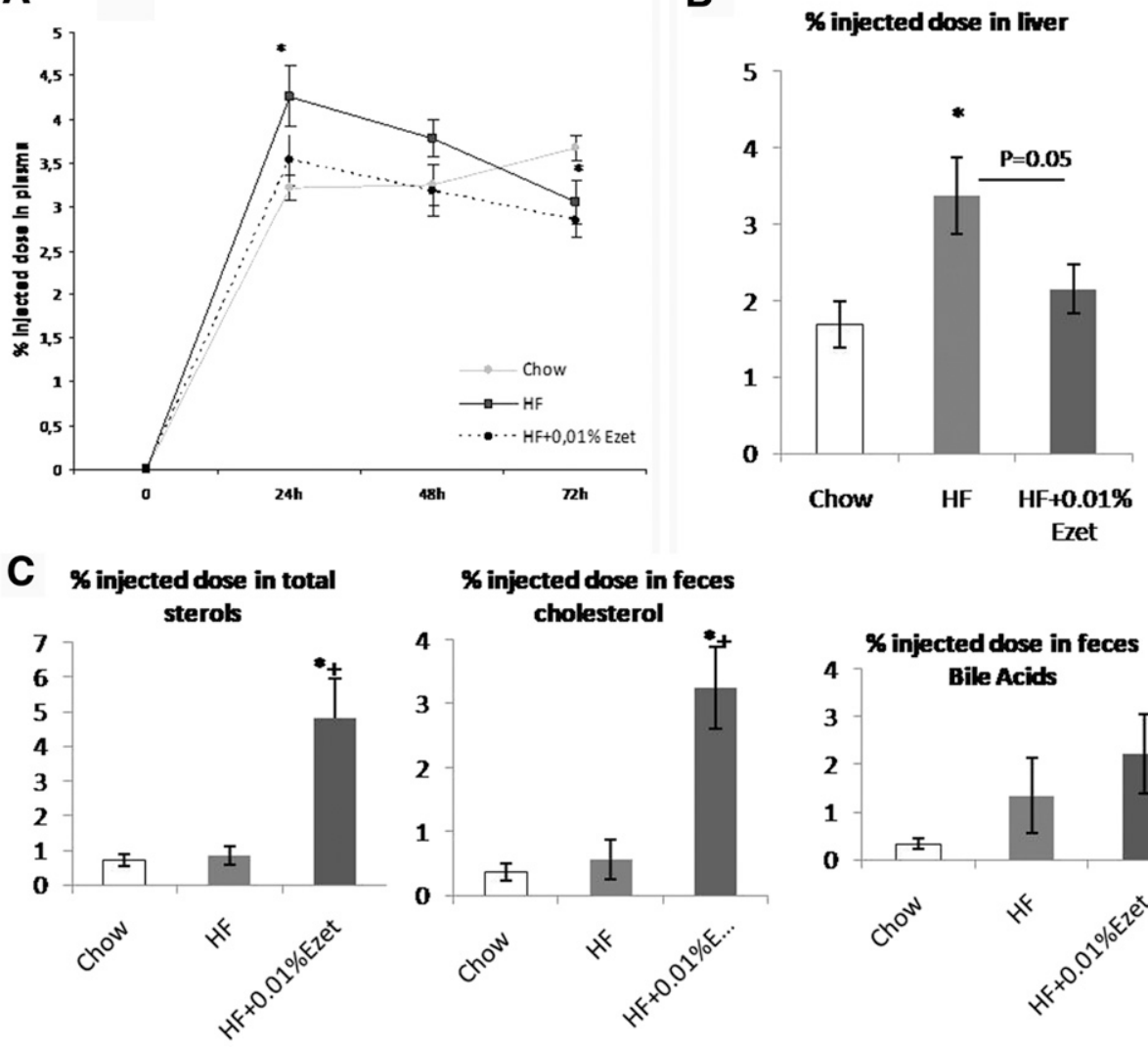

\% injected dose in feces cholesterol

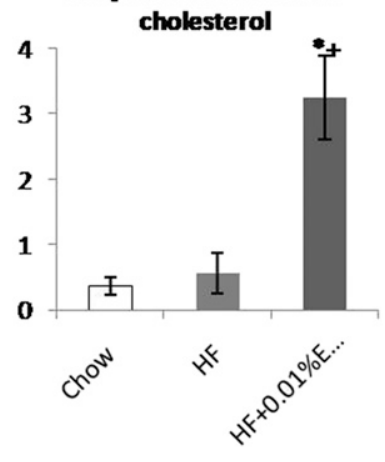

\% injected dose in feces

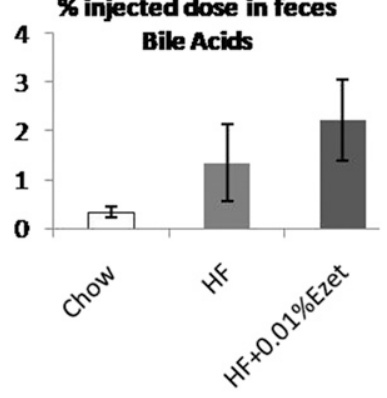

Fig. 4. Diet effect on reverse cholesterol transport after labeled macrophages injection. (A) 3H-tracer kinetic in plasma. (B) Liver $3 \mathrm{H}$-tracer at 72 hours. (C) $3 \mathrm{H}$-tracer in fecal cholesterol and bile acids. Data are expressed as percent dpm injected and mean \pm S.E.M. $(n=6$ per group; $* P<0.05$ different from control; $+P<0.05$ different from $\mathrm{HF}$ ). 
A

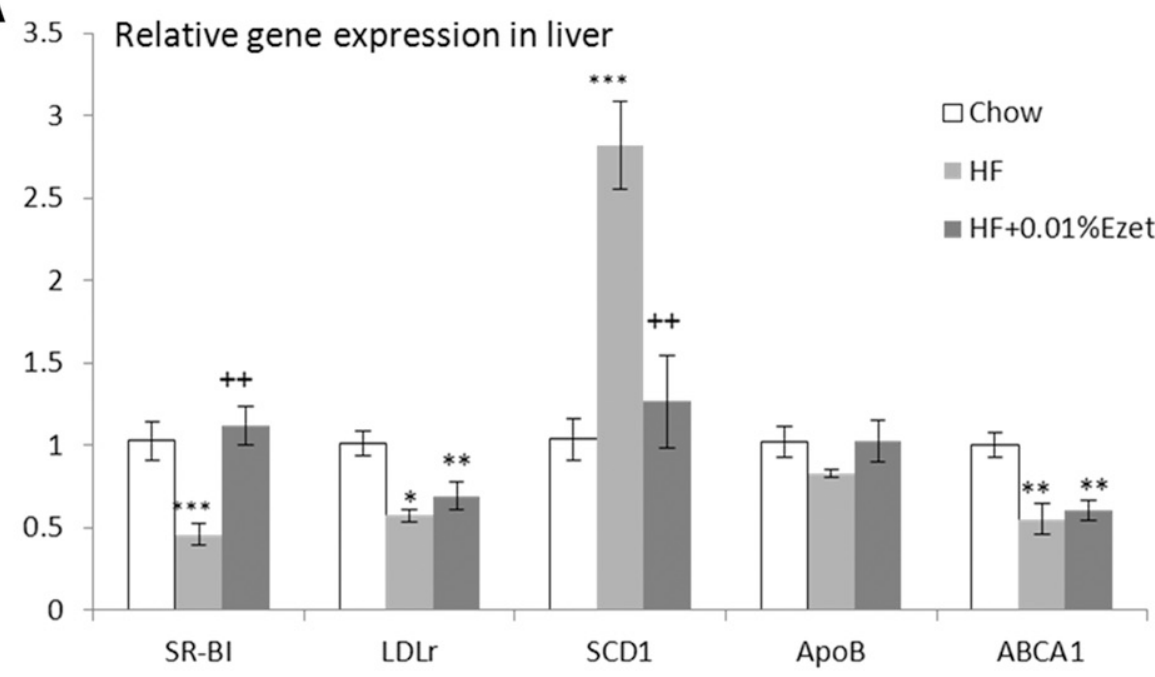

CYP7A1 relative gene expression in liver

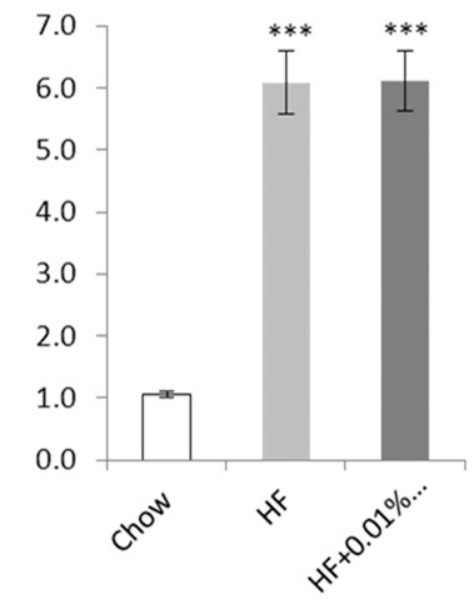

B

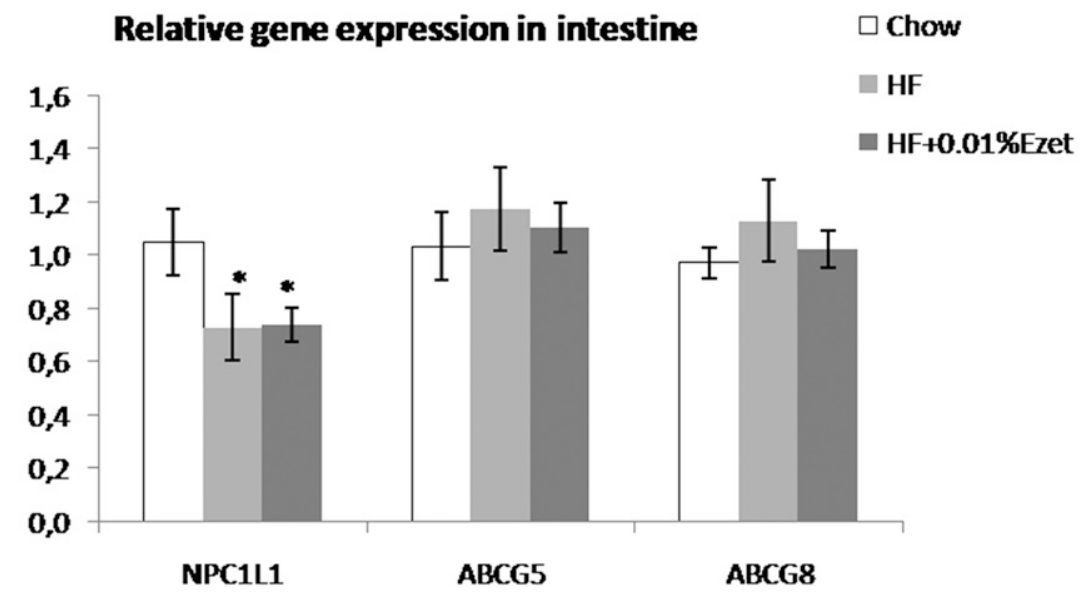

Fig. 5. Diet effect on gene expression in liver (A) and intestine (B). Mean \pm S.E.M. $(n=6$ per group; $* P<0.05$ different from control; $+P<0.05$ different from $\mathrm{HF})$.

glucose (van Heek et al., 2001). In contrast, several studies using ezetimibe described a lower fasting blood glucose associated with better glucose tolerance in hamster (Naples et al., 2012), in mice (Wang et al., 2014; Chang et al., 2015), in rat (Deushi et al., 2007), and in human (Park et al., 2011). This effect was related to the stimulation of glucagon-like peptide 1 by ezetimibe (Chang et al., 2015) or the improvement of lipid profile and hepatic insulin sensitivity (Deushi et al., 2007; Park et al., 2011). In a study focusing on obesity and diabetes, ezetimibe treatment or NPC1L1 deficiency was shown to attenuate weight gain and insulin resistance in mice fed a high-fat diet (Labonte et al., 2008). In our study, the use of high-cholesterol and high-fructose diet could explain the lack of effect on glucose metabolism compared with studies using only high-fat diet.

Interestingly, ezetimibe also improved hepatic lipid accumulation and systemic dyslipidemia induced by high-cholesterol diet. This result is consistent with others in which ezetimibe treatment improved hepatic steatosis in leptin receptor-deficient Zucker obese rats (Deushi et al., 2007; Nomura et al., 2009). Ezetimibe also reduced hepatic steatosis in mice on the methionine choline-deficient diet (Assy et al., 2006) or diets containing high amounts of cholesterol (Zheng et al., 2008; Nozaki et al., 2009). In another study focusing on the role of NPC1L1 in protein trafficking, NPC1L1 knockout mice were shown to be resistant to hepatic steatosis induced by a lithogenic diet that contains high amounts of bile acids plus cholesterol and fat (Davies et al., 2005). Fat content of the liver reflects the balance between nonesterified fatty acid flux, fatty acid oxidation, de novo lipogenesis, and VLDL secretion. Taking into consideration its primary effect, ezetimibe seemed to ameliorate liver steatosis by decreasing lipid absorption and subsequently decreased lipoprotein synthesis. On the other hand, in our study hepatic TG secretion was not affected by ezetimibe treatment despite the decrease in hepatic SCD1 gene expression observed in treated hamsters. This result is in accordance with a study in mice (Wang et al., 2014) and in obese rats (Deushi et al., 2007) treated with ezetimibe, which indicates that ezetimibe may affect clearance or uptake but not secretion of triglyceride (MacArthur et al., 2007). 
In our study as in others, ezetimibe promoted macrophage reverse cholesterol transport. We showed that using NPC1L1 inhibitor resulted in an increase in fecal cholesterol excretion. In this study, we showed that ezetimibe treatment decreased plasma cholesterol by decreasing VLDL and LDL cholesterol without affecting HDL cholesterol. These changes were associated with an increase in hepatic SR-B1 gene expression, indicating a possible higher selective uptake of HDL cholesterol. This result was not associated with change in ABCA1 gene expression nor in CETP and LCAT activity, suggesting that although ezetimibe treatment significantly increased SRB1 expression, it did not affect HDL formation. Study in mice indicated a similar result with no change in HDL cholesterol (Briand et al., 2009). Regarding non-HDL cholesterol metabolism, ezetimibe decreased LDL and VLDL plasma cholesterol, but no change was observed in LDLr gene expression, and as mentioned above, no effect was observed in CETP activity. Apolipoprotein B100 kinetic studies in men with primary hypercholesterolemia revealed that ezetimibe decreased LDL cholesterol, primarily through enhanced catabolic rates of VLDL, intermediate-density lipoprotein, and LDL, which is consistent with an upregulation of hepatic LDL receptor activity (Tremblay et al., 2006). These data suggested that ezetimibe was acting mainly in intestine by inhibiting cholesterol absorption, leading to more fecal cholesterol excretion. Higher fecal cholesterol excretion was also shown (Siri et al., 2001) in hamster under high-cholesterol diet treated with ezetimibe (Uto-Kondo et al., 2014). In this later study, bile duct ligation in hamsters abolished the enhancement of fecal excretion of macrophage-derived ${ }^{3} \mathrm{H}$-cholesterol, suggesting that ezetimibe promotes RCT by the inhibition of the cholesterol absorption, which is mainly derived from bile under ezetimibe treatment (Uto-Kondo et al., 2014). In our study, the labeled cholesterol recovered in the feces was significantly increased, whereas no change occurred in the bile acids fraction. This result was associated with no change in hepatic CYP7A1 gene expression, indicating that ezetimibe did not affect the conversion of cholesterol into bile acids. Our results are in accordance with those observed in mice (Briand et al., 2009) and hamsters (Uto-Kondo et al., 2014).

As in our study, Repa et al. (2004) reported that NPC1L1 expression was lower in the intestine of mice fed a cholesterolenriched diet compared with those on chow one; in contrast, Field et al. (2004) showed that intestinal NPC1L1 expression in hamsters was much less sensitive to a cholesterol-enriched diet. In the present study, ezetimibe did not affect ABCA1, ABCG5, ABCG8, and NPC1L1 relative gene expression; similarly, a study in hamster measured protein expression of ABCG5, ABCG8, and NPC1L1 showed no change with ezetimibe treatment (During et al., 2005; Naples et al., 2012), whereas these genes were described to be downregulated by ezetimibe in Caco-2 cells (During et al., 2005). A recent study showed that ezetimibe may not only prevent atherosclerosis by inhibiting cholesterol absorption in the intestine but also by increasing the expression of apolipoprotein A-II in hepatocytes (Yan et al., 2019). Study in human showed in accordance with this hypothesis that ezetimibe increased the efficiency of reverse cholesterol transport from rapidly mixing plasma and tissue cholesterol pools into the stool (Lin et al., 2017). Although we did not show a change in ABCG5, ABCG8, or NPC1L1 expression, we cannot exclude a potential effect of ezetimibe on transintestinal cholesterol excretion. The atheroprotective effect of ezetimibe may result in stimulation in at least one of transintestinal cholesterol excretion pathways: 1) excretion into the intestinal lumen mainly via ABCG5/8, 2) cholesterol absorption from the intestine by Niemann-Pick C1-like protein 1,3) the uptake of plasma lipoproteins by enterocytes at the basolateral membrane, or 4) the excretion of cholesterol in chylomicrons into the lymph (Reeskamp et al., 2018). The mechanism by which ezetimibe controlled cholesterol absorption and especially how it affects the expression of genes involved in cholesterol absorption remain not clear, and the mechanism implying the role of ezetimibe as NPC1L1 blocker remains the most plausible.

In conclusion, ezetimibe exhibited an atheroprotective effect by enhancing RCT in hamster and decreasing LDL cholesterol. Our findings also showed a hepatoprotective effect of ezetimibe by decreasing hepatic fat content. Despite these interesting observations, the mechanism by which NPC1L1 deficiency or ezetimibe treatment alleviates hepatic steatosis, insulin resistance, and obesity remains largely unexplored and needs more investigation in animal model and in human.

\section{Authorship Contributions}

Participated in research design: Briand, Sulpice, Ouguerram. Conducted experiments: Kasbi-Chadli, Treguier.

Performed data analysis: Kasbi-Chadli, Treguier.

Wrote or contributed to the writing of the manuscript: KasbiChadli,, Ouguerram.

\section{References}

Altmann SW, Davis HR Jr., Zhu LJ, Yao X, Hoos LM, Tetzloff G, Iyer SP, Maguire M, Golovko A, Zeng M, et al. (2004) Niemann-Pick C1 Like 1 protein is critical for intestinal cholesterol absorption. Science 303:1201-1204.

Assy N, Grozovski M, Bersudsky I, Szvalb S, and Hussein O (2006) Effect of insulinsensitizing agents in combination with ezetimibe, and valsartan in rats with nonalcoholic fatty liver disease. World J Gastroenterol 12:4369-4376.

Bernard DW, Rodriguez A, Rothblat GH, and Glick JM (1991) cAMP stimulates cholesteryl ester clearance to high density lipoproteins in J7774 macrophages. $J$ Biol Chem 266:710-716.

Briand F (2010) The use of dyslipidemic hamsters to evaluate drug-induced alterations in reverse cholesterol transport. Curr Opin Investig Drugs 11:289-297.

Briand F, Naik SU, Fuki I, Millar JS, Macphee C, Walker M, Billheimer J, Rothblat G, and Rader DJ (2009) Both the peroxisome proliferator-activated receptor delta agonist, GW0742, and ezetimibe promote reverse cholesterol transport in mice by reducing intestinal reabsorption of HDL-derived cholesterol. Clin Transl Sci 2: 127-133.

Castro-Perez J, Briand F, Gagen K, Wang SP, Chen Y, McLaren DG, Shah V, Vreeken RJ, Hankemeier T, Sulpice T, et al. (2011) Anacetrapib promotes reverse cholesterol transport and bulk cholesterol excretion in Syrian golden hamsters. $J$ Lipid Res 52:1965-1973.

Chang E, Kim L, Choi JM, Park SE, Rhee EJ, Lee WY, Oh KW, Park SW, Park DI, and Park CY (2015) Ezetimibe stimulates intestinal glucagon-like peptide 1 secretion via the MEK/ERK pathway rather than dipeptidyl peptidase 4 inhibition. Metabolism 64:633-641.

Cuchel M and Rader DJ (2006) Macrophage reverse cholesterol transport: key to the regression of atherosclerosis? Circulation 113:2548-2555.

Davies JP, Scott C, Oishi K, Liapis A, and Ioannou YA (2005) Inactivation of NPC1L1 causes multiple lipid transport defects and protects against diet-induced hypercholesterolemia. J Biol Chem 280:12710-12720.

Deushi M, Nomura M, Kawakami A, Haraguchi M, Ito M, Okazaki M, Ishii H, and Yoshida M (2007) Ezetimibe improves liver steatosis and insulin resistance in obese rat model of metabolic syndrome. FEBS Lett 581:5664-5670.

During A, Dawson HD, and Harrison EH (2005) Carotenoid transport is decreased and expression of the lipid transporters SR-BI, NPC1L1, and ABCA1 is downregulated in Caco-2 cells treated with ezetimibe. J Nutr 135:2305-2312.

Field FJ, Born E, and Mathur SN (2004) Stanol esters decrease plasma cholesterol independently of intestinal ABC sterol transporters and Niemann-Pick C1-like 1 protein gene expression. J Lipid Res 45:2252-2259.

Folch J, Lees M, and Sloane Stanley GH (1957) A simple method for the isolation and purification of total lipides from animal tissues. J Biol Chem 226:497-509.

Garcia-Calvo M, Lisnock J, Bull HG, Hawes BE, Burnett DA, Braun MP, Crona JH, Davis HR Jr., Dean DC, Detmers PA, et al. (2005) The target of ezetimibe is Niemann-Pick C1-Like 1 (NPC1L1). Proc Natl Acad Sci USA 102:8132-8137.

Ginsberg HN, Zhang YL, and Hernandez-Ono A (2006) Metabolic syndrome: focus on dyslipidemia. Obesity (Silver Spring) 14 (Suppl 1):41S-49S.

Jacobson TA, Ito MK, Maki KC, Orringer CE, Bays HE, Jones PH, McKenney JM Grundy SM, Gill EA, Wild RA, et al. (2015) National lipid association recommendations 
for patient-centered management of dyslipidemia: part 1--full report. J Clin Lipidol 9: $129-169$.

Kasbi Chadli F, Nazih H, Krempf M, Nguyen P, and Ouguerram K (2013) Omega 3 fatty acids promote macrophage reverse cholesterol transport in hamster fed high fat diet. PLoS One 8:e61109.

Labonté ED, Camarota LM, Rojas JC, Jandacek RJ, Gilham DE, Davies JP, Ioannou YA, Tso P, Hui DY, and Howles PN (2008) Reduced absorption of saturated fatty acids and resistance to diet-induced obesity and diabetes by ezetimibe-treated and Npc1l1-/- mice. Am J Physiol Gastrointest Liver Physiol 295:G776-G783.

Lin X, Racette SB, Ma L, Wallendorf M, and Ostlund RE Jr. (2017) Ezetimibe increases endogenous cholesterol excretion in humans. Arterioscler Thromb Vasc Biol 37:990-996.

MacArthur JM, Bishop JR, Stanford KI, Wang L, Bensadoun A, Witztum JL, and Esko JD (2007) Liver heparan sulfate proteoglycans mediate clearance of triglyceride-rich lipoproteins independently of LDL receptor family members. $J$ Clin Invest 117:153-164.

Mazzone T and Reardon C (1994) Expression of heterologous human apolipoprotein E by J774 macrophages enhances cholesterol efflux to HDL3. J Lipid Res $\mathbf{3 5}$ 1345-1353.

Millar JS, Maugeais C, Fuki IV, and Rader DJ (2002) Normal production rate of apolipoprotein B in LDL receptor-deficient mice. Arterioscler Thromb Vasc Biol 22: 989-994.

Nagasaki T and Akanuma Y (1977) A new colorimetric method for the determination of plasma lecithin-cholesterol acyltransferase activity. Clin Chim Acta 75:371-375.

Naples M, Baker C, Lino M, Iqbal J, Hussain MM, and Adeli K (2012) Ezetimibe ameliorates intestinal chylomicron overproduction and improves glucose tolerance in a diet-induced hamster model of insulin resistance. Am J Physiol Gastrointest Liver Physiol 302:G1043-G1052.

Nomura M, Ishii H, Kawakami A, and Yoshida M (2009) Inhibition of hepatic Niemann-Pick C1-like 1 improves hepatic insulin resistance. Am J Physiol Endocrinol Metab 297:E1030-E1038.

Nozaki Y, Fujita K, Yoneda M, Wada K, Shinohara Y, Takahashi H, Kirikoshi H, Inamori M, Kubota K, Saito S, et al. (2009) Long-term combination therapy of ezetimibe and acarbose for non-alcoholic fatty liver disease. $J$ Hepatol 51: $548-556$.

Ouchi Y, Sasaki J, Arai H, Yokote K, Harada K, Katayama Y, Urabe T, Uchida Y, Hayashi M, Yokota N, et al. (2019) Ezetimibe lipid-lowering trial on prevention of atherosclerotic cardiovascular disease in 75 or older (EWTOPIA 75): a randomized, controlled trial. Circulation 140:992-1003.

Park H, Shima T, Yamaguchi K, Mitsuyoshi H, Minami M, Yasui K, Itoh Y, Yoshikawa T, Fukui M, Hasegawa G, et al. (2011) Efficacy of long-term ezetimibe therapy in patients with nonalcoholic fatty liver disease. $J$ Gastroenterol 46 101-107.

Reeskamp LF, Meessen ECE, and Groen AK (2018) Transintestinal cholesterol excretion in humans. Curr Opin Lipidol 29:10-17.
Repa JJ, Buhman KK, Farese RV Jr., Dietschy JM, and Turley SD (2004) ACAT2 deficiency limits cholesterol absorption in the cholesterol-fed mouse: impact on hepatic cholesterol homeostasis. Hepatology 40:1088-1097.

Schotz MC, Scanu A, and Page IH (1957) Effect of triton on lipoprotein lipase of rat plasma. Am J Physiol 188:399-402.

Sehayek E and Hazen SL (2008) Cholesterol absorption from the intestine is a major determinant of reverse cholesterol transport from peripheral tissue macrophages. Arterioscler Thromb Vasc Biol 28:1296-1297.

Siri P, Candela N, Zhang YL, Ko C, Eusufzai S, Ginsberg HN, and Huang LS (2001) Post-transcriptional stimulation of the assembly and secretion of triglyceride-rich apolipoprotein B lipoproteins in a mouse with selective deficiency of brown adipose tissue, obesity, and insulin resistance. J Biol Chem 276:46064-46072.

Tchoua U, D’Souza W, Mukhamedova N, Blum D, Niesor E, Mizrahi J, Maugeais C, and Sviridov D (2008) The effect of cholesteryl ester transfer protein overexpression and inhibition on reverse cholesterol transport. Cardiovasc Res 77: 732-739.

Tréguier M, Briand F, Boubacar A, André A, Magot T, Nguyen P, Krempf M, Sulpice $\mathrm{T}$, and Ouguerram K (2011) Diet-induced dyslipidemia impairs reverse cholesterol transport in hamsters. Eur J Clin Invest 41:921-928.

Tremblay AJ, Lamarche B, Cohn JS, Hogue JC, and Couture P (2006) Effect of ezetimibe on the in vivo kinetics of apoB-48 and apoB-100 in men with primary hypercholesterolemia. Arterioscler Thromb Vasc Biol 26:1101-1106.

Uto-Kondo H, Ayaori M, Sotherden GM, Nakaya K, Sasaki M, Yogo M, Komatsu T, Takiguchi S, Yakushiji E, Ogura M, et al. (2014) Ezetimibe enhances macrophage reverse cholesterol transport in hamsters: contribution of hepato-biliary pathway. Biochim Biophys Acta 1841:1247-1255.

van Heek M, Austin TM, Farley C, Cook JA, Tetzloff GG, and Davis HR (2001) Ezetimibe, a potent cholesterol absorption inhibitor, normalizes combined dyslipidemia in obese hyperinsulinemic hamsters. Diabetes 50:1330-1335.

Wang X, Ren Q, Wu T, Guo Y, Liang Y, and Liu S (2014) Ezetimibe prevents the development of non-alcoholic fatty liver disease induced by high-fat diet in C57BL/ 6J mice. Mol Med Rep 10:2917-2923.

Yan Y, He F, Li Z, Xu R, Li T, Su J, Liu X, Zhao M, and Wu W (2019) The important role of apolipoprotein A-II in ezetimibe driven reduction of high cholesterol dietinduced atherosclerosis. Atherosclerosis 280:99-108.

Zheng S, Hoos L, Cook J, Tetzloff G, Davis H Jr., van Heek M, and Hwa JJ (2008) Ezetimibe improves high fat and cholesterol diet-induced non-alcoholic fatty liver disease in mice. Eur $J$ Pharmacol 584:118-124.

Address correspondence to: Khadija Ouguerram, CHU Hôtel-Dieu, F-44 000 Nantes, France; CRNH, Western Human Nutrition Research Center, CHU Hôtel-Dieu, F-44093 Nantes, France. E-mail: khadija.ouguerram@univnantes.fr 